\title{
Unitary Detector DPC Imaging with Multiscale Capabilities for Analysis of Local Magnetic Field of Nanomaterials.
}

\author{
Yurii P. Ivanov ${ }^{1}$, Sergei Lopatin ${ }^{1}$, Jurgen $\operatorname{Kosel}^{1}$ and Andrey Chuvilin ${ }^{2,3}$. \\ 1. King Abdullah University of Science \& Technology, Thuwal, Makkah 23955, Saudi Arabia \\ 2. CIC nanoGUNE, Av. de Tolosa 76, E-20018, Donostia-San Sebastian, Spain \\ 3. IKERBASQUE, Basque Foundation for Science, Maria Diaz de Haro 3, 48013 Bilbao, Spain.
}

To advance with creation of novel nano-electronic devices there is a need to understand electrons behavior in a wide range of materials with dimensions reduced down to nanometer scale. It is an important task for industries dealing with magnetic memories [1], light-emitting, photovoltaic or multiferroic devices [2]. A key role for development of such devices is the nanoscale characterization of the local electro-magnetic fields in the materials [3].

Out of the many Transmission Electron Microscopy (TEM) techniques the Differential Phase Contrast DPC [4] method is the most useful for fast switching between studying of objects of about $10 \mu \mathrm{m}$ down to a few $\mathrm{nm}$ in size (multiscale imaging). But the conventional DPC requires specially designed position sensitive detector(s) (split, segmented, multi-sectored, etc) and costly hardware solutions, thus significantly limiting the wide use of the method.

We report a generalization of the DPC imaging method to extend the capabilities of the majority of existing TEM systems (without modifications or upgrades) towards multiscale characterization of local electro-magnetic properties of nanomaterials. The usability of our method both at micro- and nano- scale is tested on the investigation of $80 \mathrm{~nm}$ diameter cylindrical $\mathrm{Co} / \mathrm{Ni}$ nanowires with a high length to diameter ratio - promising candidates for 3D magnetic memory devices.

The conventional DPC typically requires a detector divided into 4 quadrants. Physically it is equivalent to 4 unitary (non-segmented) detectors in positions A-B-C-D (Fig.1a, b). Alternatively we can use just one unitary detector but physically move it in a circle (with consecutive steps of $90^{\circ}$ ) to four positions (A-B-C-D) equidistant from the zero-field diffraction disc (Fig.1a). Recording signals at each position we can collect (in series) the set of DPC images.

The drawback of such approach is that 4 images are acquired not simultaneously but in series. On the other hand, the detector positions can be voluntarily chosen in such a way that axis A-C or B-D is in line with a preferential direction of the sample. This is especially important for sample flipping (as normally required for magnetic field visualization). In this case one can redefine detector positions in line with TEM sample instead of rigorous repositioning the sample in a holder. The biggest advantage, though, is that the new scheme requires only one, unitary detector and, in principle, any STEM detector physically present on the microscope can be used.

Since for most TEM systems the detector position is not flexible, it is more practical to reposition consecutively the diffraction disc, this time going in a circle around a fixed unitary detector: Fig. 1c, d. With the same equidistance between the zero-field diffraction disc and the detector (Fig.1c), the positions $A^{*}-B^{*}-C^{*}-D^{*}$ in both Fig.1c and $d$ are equivalent to positions A-B-C-D in Fig.1a and b. It is this approach we use for DPC imaging in a combination with virtual bright field (VBF) detector. 
Our method demonstrates high sensitivity to the local magnetic fields, provides a very large field of view, a few nanometers spatial resolution and in-focus condition. The application of our approach allows us to visualize reliably the magnetic structure of multisegmented cylindrical $\mathrm{Co} / \mathrm{Ni}$ nanowire (Fig. 2), quantify magnetization of different segments and image vortex domain wall pinned at the $\mathrm{Co} / \mathrm{Ni}$ interface (the middle of the fig. $2 \mathrm{~b}, \mathrm{c}$ ).

\section{References:}

[1] R. Stamps et al, J. Phys. D: Appl. Phys. 47 (2014), p. 333001.

[2] J. F. Scott, Science 315 (2007), p. 954.

[3] W. Jiang et al, Science 349 (2015), p. 283.

[4] H. Rose, Ultramicroscopy 2 (1977), p. 251.

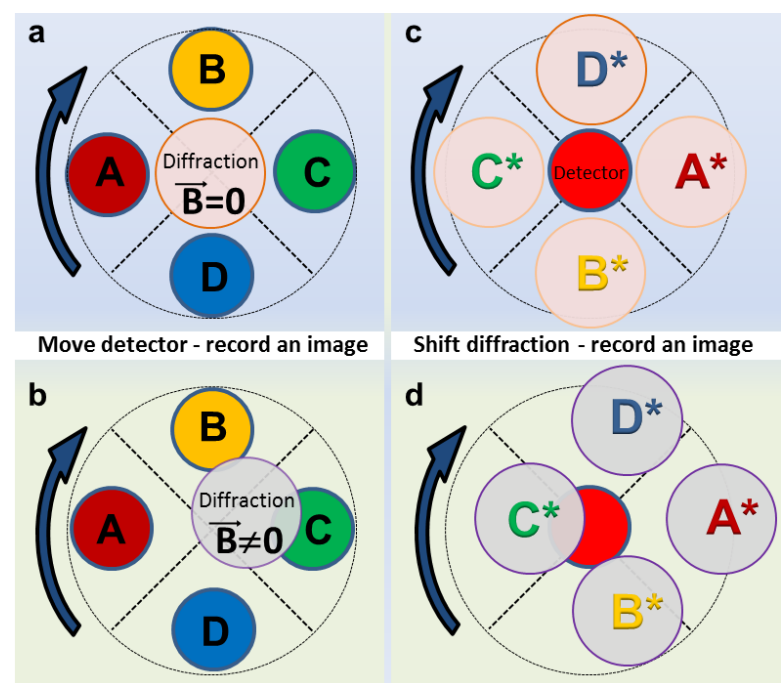

Figure 1. Transition from a conventional four-quadrant detector to a unitary detector DPC imaging. a, b, Four unitary detectors or one detector repositioning around diffraction disc. c, d, Diffraction disc shift around the unitary detector

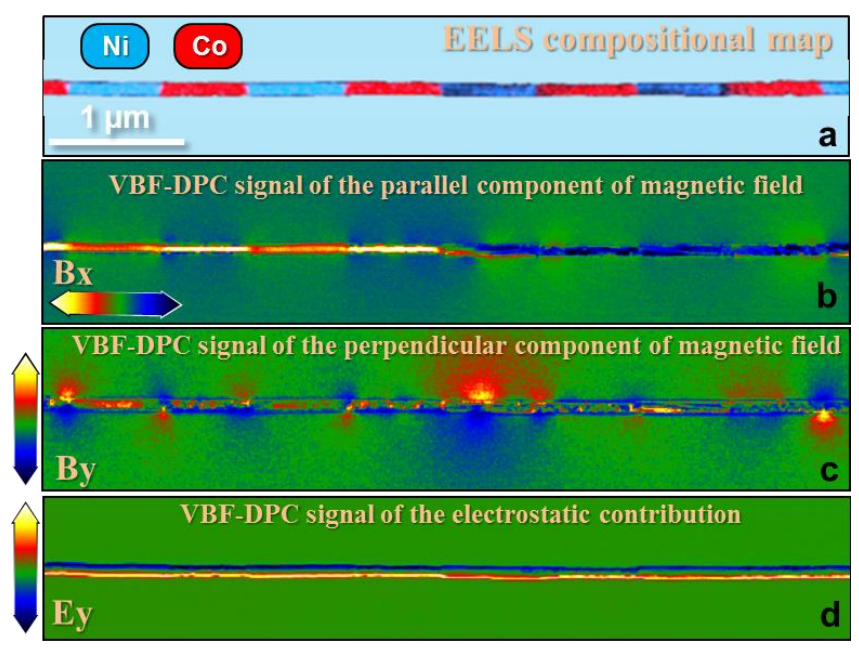

Figure. 2. Characterization of multisegment $\mathrm{Co} / \mathrm{Ni}$ nanowire. a, Variation of composition obtained by EELS. b, c The magnetic field components parallel Bx and perpendicular By to the nanowire length. d, The electrostatic contribution perpendicular Ey to the nanowire length. 